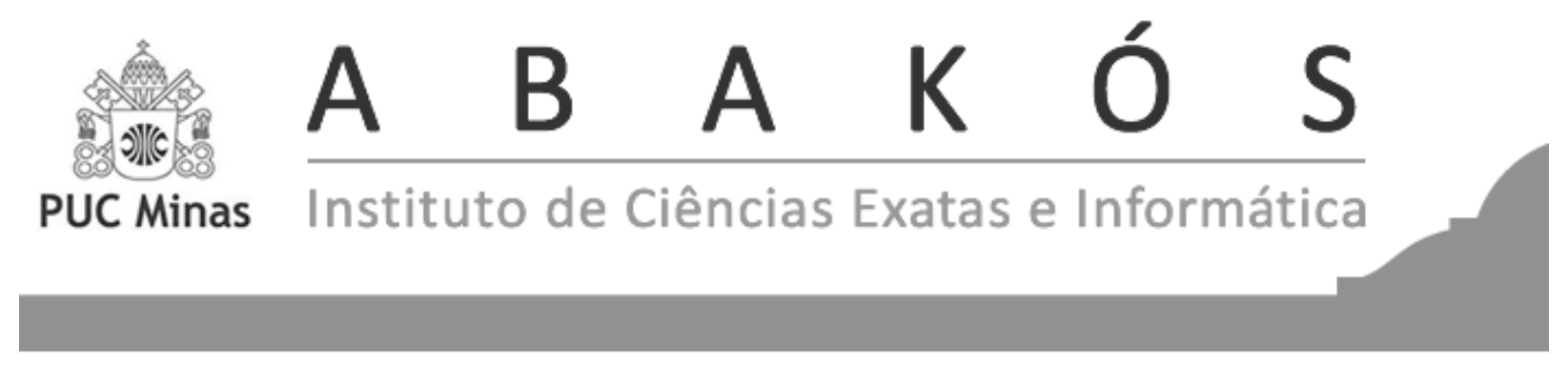

cc) (†)

\title{
Modelagem do Tempo de Falha de um Transístor*
}

Modelling the Failure Time of a Transistor

\author{
Marcelo Pereira Fernandes Júnior ${ }^{1}$ \\ Marcos Henrique Palma Lopes ${ }^{2}$ \\ Paulo César de Resende Andrade ${ }^{3}$
}

\begin{abstract}
Resumo
A aplicação de técnicas de confiabilidade permite descrever o comportamento de falhas em equipamentos. O presente trabalho tem como objetivo modelar o tempo de vida de um transístor por meio da análise de confiabilidade paramétrica. O método de pesquisa utilizado consistiu no estudo de caso, com a coleta dos tempos de falhas de 24 transístores. Métodos gráficos e analíticos foram utilizados, por meio do software ProConf, para verificar que a distribuição que melhor se ajusta aos dados é a distribuição Weibull. As funções de confiabilidade e de risco foram apresentadas. O risco crescente e a confiabilidade decrescente, associados ao fato do parâmetro de forma $\gamma$ ser maior que um, indicam um desgaste do equipamento. Isso corresponde ao início da mortalidade senil, fase final de sua vida útil.
\end{abstract}

Palavras-chave: Confiabilidade. Curva da banheira. Mortalidade senil. Distribuição de Weibull.

\footnotetext{
* Submetido em 29/01/2019 - Aceito em 06/09/2019

${ }^{1}$ Bacharelando em Ciência e Tecnologia, pela Universidade dos Vales do Jequitinhonha e Mucuri (UFVJM), Brasilmarcelo152007@hotmail.com

${ }^{2}$ Bacharelando em Ciência e Tecnologia, pela Universidade dos Vales do Jequitinhonha e Mucuri (UFVJM), Brasilmarcoshpalma@gmail.com

${ }^{3}$ Professor do Instituto de Ciência e Tecnologia da Universidade dos Vales do Jequitinhonha e Mucuri (UFVJM), Brasil-pceandrade@gmail.com
} 


\begin{abstract}
The present work aims to model the life span of a transistor through parametric reliability analysis. The application of reliability techniques allows the description of the behavior of equipment failures. The research method consisted of a case study based on the collected failure time of 24 transistors. Graphical and analytical methods, through the ProConf software, were used to verify that the Weibull distribution is the one that best fits the data. The reliability and risk functions are presented. The increasing risk and decreasing reliability, associated with the fact that the parameter $\gamma$ is greater than one, indicates equipment wear. This corresponds to the onset of senile mortality, the final phase of its useful life.
\end{abstract}

Keywords: Curve of the bathtub. Reliability. Senile Mortality. Weibull distribution. 


\section{INTRODUÇÃO}

O desenvolvimento tecnológico provocou uma revolução no setor econômico com o aumento significativo da concorrência entre as empresas. Com o advento da economia globalizada, observou-se um aumento na demanda por produtos e sistemas de melhores desempenhos e custos competitivos. Diante disso, as organizações procuram evitar falhas nos equipamentos ou produtos. As falhas em componentes ou sistemas causam efeitos que vão desde inconveniências menores até impactos severos no mercado, gerando perdas econômicas significativas (MENDES, 2011).

A NBR ISO 8402 (ABNT, 1994) define que a confiabilidade está relacionada à qualidade, disponibilidade, mantenabilidade, segurança e confiança. O custo anual de falta de confiabilidade é alto, o que levou a uma preocupação maior com o conhecimento mais profundo das características técnicas, operacionais, ambientais, de falhas e de manutenção dos equipamentos. Em busca de melhores produtos e serviços as empresas têm procurado ferramentas, para a melhoria da confiabilidade e segurança, visando minimizar as falhas (SILVA, 2012).

A coleta de dados passou a ser uma atividade a longo prazo para maior conhecimento e diminuição dos custos para manutenção e troca de equipamentos (KARDEC; NASCIF, 2009). Consequentemente, surgiu a necessidade de redução na probabilidade de falhas em produtos, o que resultou em uma ênfase crescente em confiabilidade. A análise de confiabilidade é uma metodologia utilizada para descrever o comportamento dessas falhas (FOGLIATO; RIBEIRO, 2009). Piazza (2000) completa afirmando que uma das qualidades exigidas pelos clientes de um produto é a confiabilidade em que é fabricado.

De uma forma genérica, confiabilidade pode ser definida como a possibilidade de um componente, equipamento, ou sistema desempenhar satisfatoriamente a função requerida, sob condições de operação estabelecidas, por um período de tempo predeterminado, sem apresentar falhas. Confiabilidade é representada em termos de uma probabilidade (LEEMIS, 1995). Algumas distribuições podem ser utilizadas para representar a probabilidade de falha, possibilitando determinar a partir de que momento o produto sob análise é considerado com desempenho abaixo daquele apontado como aceitável e, portanto, considerado com a ocorrência de falha (HAVIARAS, 2005).

A análise de confiabilidade possibilita, por meio de estimativa, caracterizar seus comportamentos, da probabilidade de falha e da taxa de falha em relação ao tempo de um componente, equipamento ou sistema. O comportamento de determinado produto com relação à falha é melhor compreendido pelo estudo de tempo de falha e exame do comportamento de sua taxa de falha (COLOSIMO; FREITAS, 1997). Por tempo até a falha, entende-se o tempo transcorrido desde o momento em que a unidade é colocada em operação até que ocorra sua primeira falha.

A análise de confiabilidade de um sistema é realizada observando o histórico de falhas de um determinado componente (GAIO, 2016). Para isso, é necessário fazer a modelagem de dados históricos de tempos até as falhas em distribuições de probabilidades. As distribuições de probabilidade que frequentemente são mais utilizadas para descrever os tempos até a falha de 
componentes e sistemas são: Exponencial, Gama, Lognormal e Weibull (HAVIARAS, 2005).

A distribuição Exponencial é relevante em estudos de confiabilidade por ser a única distribuição contínua com função do risco constante ao longo de sua vida útil. É o que ocorre com componentes eletrônicos, tais como capacitores, transistores, resistores e circuitos integrados (LEEMIS, 1995). Sua função de distribuição de probabilidade acumulada corresponde a $\mathrm{F}(\mathrm{t})=1-\mathrm{e}^{-\lambda \mathrm{t}}$ sua função de densidade de probabilidade $\mathrm{f}(\mathrm{t})=\lambda \mathrm{e}^{-\lambda \mathrm{t}}$, com função de risco $\mathrm{h}(\mathrm{t})=\lambda$ e função de confiabilidade $\mathrm{R}(\mathrm{t})=\mathrm{e}^{-\lambda \mathrm{t}}$; sendo $\lambda$ seu parâmetro.

A distribuição Gama é apropriada para a descrição de tempos de falhas de sistemas associadas a certo número de sub-falhas com taxa de ocorrência constante, ou de um componente cuja falha somente é efetivada após um certo número de estágios (ELSAYED, 1996).

A Lognormal é uma distribuição amplamente utilizada em ajustes de dados por apresentar uma grande quantidade de formatos, de acordo com seus parâmetros. É muito utilizada na modelagem de tempos até reparo em unidades reparáveis (FOGLIATO; RIBEIRO, 2009). A taxa de risco desse modelo apresenta comportamentos diversos, de acordo com os valores dos parâmetros $\mu$ e $\sigma$.

A distribuição Weibull é uma das mais importantes distribuições em modelos de confiabilidade devido à flexibilidade e capacidade de representação de amostras de tempos até a falha com comportamentos distintos (LAFRAIA, 2001). Segundo Khan et al. (2008), essa distribuição é bastante adequada para testes de vida e na modelagem de componentes mecânicos ou elétricos. Sua função de distribuição de probabilidade acumulada corresponde a $F(t)=1-e^{-\left(\frac{t}{\theta}\right)^{\gamma}}$, sua função de densidade de probabilidade é dada por $\mathrm{f}(\mathrm{t})=\frac{\gamma}{\theta^{\gamma}} \mathrm{t}^{\gamma-1} \mathrm{e}^{-\left(\frac{t}{\theta}\right)^{\gamma}}$, com função de risco $\mathrm{h}(\mathrm{t})=\frac{\gamma}{\theta \gamma} \mathrm{t}^{\gamma-1}$ e função de confiabilidade $\mathrm{R}(\mathrm{t})=\mathrm{e}^{-\left(\frac{t}{\theta}\right)^{\gamma}}$. Os parâmetros $\theta$ e $\gamma$ são respectivamente denominados parâmetro de escala (ou característica de vida) e parâmetro de forma.

As funções mais utilizadas para análise da confiabilidade são as funções de confiabilidade e de risco. A função de confiabilidade $\mathrm{R}(\mathrm{t})$ é a probabilidade de um sistema cumprir uma missão preestabelecida, com uma duração determinada, sem falhas. A taxa de risco ou taxa de falha $\mathrm{h}(\mathrm{t})$ é considerada como sendo a quantidade de risco relacionada a uma unidade do tempo t, consequentemente sendo dada em termos de falha por unidade de tempo. O tempo médio até a falha (Mean Time To Failure - MTTF) define uma média de todos os tempos mensurados até ocorrer uma falha na unidade observada em questão (LEEMIS, 1995).

A modelagem dos tempos até a falha é, portanto, central em estudos de confiabilidade (FOGLIATO; RIBEIRO, 2009). Vários estudos foram desenvolvidos com comprovadas adequações a várias situações práticas (BRANDÃO; ANDRADE, 2018).

O presente trabalho tem por objetivo modelar o tempo de vida em horas de um transístor, utilizando os conceitos de análise de confiabilidade paramétrica. 


\section{MATERIAL E MÉTODOS}

O presente trabalho é um estudo de caso de um transístor, de um mesmo modelo de determinada marca. O transístor foi descoberto meio que ao acaso, nas quais diferentes materiais foram unidos produzindo estruturas com propriedades eletrônicas e ópticas. Substituiu a válvula eletrônica e tornou-se a incontestável potencialidade dos semicondutores, provocando um grande avanço na eletrônica (CHIQUITO; JúNIOR, 1998). De maneira genérica, é um dispositivo eletrônico que tem a capacidade de controlar e amplificar sinais elétricos, parecido com as antigas válvulas eletrônicas (CRUZ; PACHECO, 2004).

Os dados foram obtidos a partir de testes realizados por uma determinada empresa da área de eletrônica, cujo nome não pode ser revelado, a pedido da mesma. Os transístores m testados em condições normais de uso e o tempo de uso foi medido a partir de sua instalação. Foram feitos testes em 24 unidades que falharam e os tempos até as falhas (em horas) estão apresentados na Tabela 1.

Tabela 1 - Tempos até a falha (horas)

\begin{tabular}{c|c|c|c|c|c|c|c}
\hline \multicolumn{7}{c}{ Tempos até a falha (horas) } \\
\hline 1880 & 2310 & 530 & 580 & 680 & 440 & 480 & 480 \\
\hline 920 & 930 & 780 & 820 & 840 & 710 & 740 & 1370 \\
\hline 260 & 350 & 420 & 1050 & 1060 & 1270 & 1340 & 1070 \\
\hline
\end{tabular}

Fonte: Criada pelos autores com dados fornecidos pela empresa (2019).

A análise de confiabilidade dos dados disponibilizados foi realizada no software ProConf (FRITSCH; RIBEIRO, 1998), um programa computacional que ajusta as distribuições de tempos de falha por meio de métodos gráficos e métodos analíticos. Os métodos gráficos utilizados para comparar as curvas das distribuições de probabilidade, e verificar o modelo que apresenta melhor aderência aos dados amostrais foram os histogramas de frequência das falhas e os papéis de probabilidades.

Os testes de aderência Qui-Quadrado $\left(\mathrm{X}^{2}\right)$ e Kolmogorov-Smirnov (K-S) foram utilizados para testar a hipótese nula de que as distribuições: Exponencial, Gama, Lognormal, Normal e Weibull se ajustam aos dados em estudo. O software informa o valor-p e aponta quais distribuições não podem ser rejeitadas (FRITSCH; RIBEIRO, 1998). A validação é dada se o valor-p do teste é maior nível de significância adotado (5\%) em ambos os testes de aderência. Quando o mesmo é maior a $5 \%$ em ambos os testes, a distribuição não pode ser rejeitada, o que significa que poderá ser utilizada na modelagem. Caso mais do que uma distribuição não possa ser rejeitada, deve-se justificar a escolha por uma delas por fundamentação teórica.

Além disso, foram estimados os parâmetros da função distribuição de probabilidade que melhor modela o conjunto de dados em estudo, utilizando o método da máxima verossimilhança (MMV). O MMV considera que a melhor estimativa possível para o parâmetro é o valor que maximiza a função de verossimilhança obtida a partir dos dados amostrais. Também são apresentadas as representações gráficas das funções de confiabilidade R(t) e de risco ou taxa de 
falha $h(t)$.

O comportamento da taxa de falha de um equipamento ao longo do tempo pode ser analisado pela curva da banheira, que representa genericamente a função de risco $h(t)$ ao longo do ciclo de vida. A curva da banheira, representada na Figura 1, apresenta três períodos característicos de vida de componentes e equipamentos: mortalidade infantil, fase de maturidade, e mortalidade senil (LAFRAIA, 2001; SELLITTO, 2005).

Figura 1 - Curva da banheira e o ciclo de vida dos equipamentos

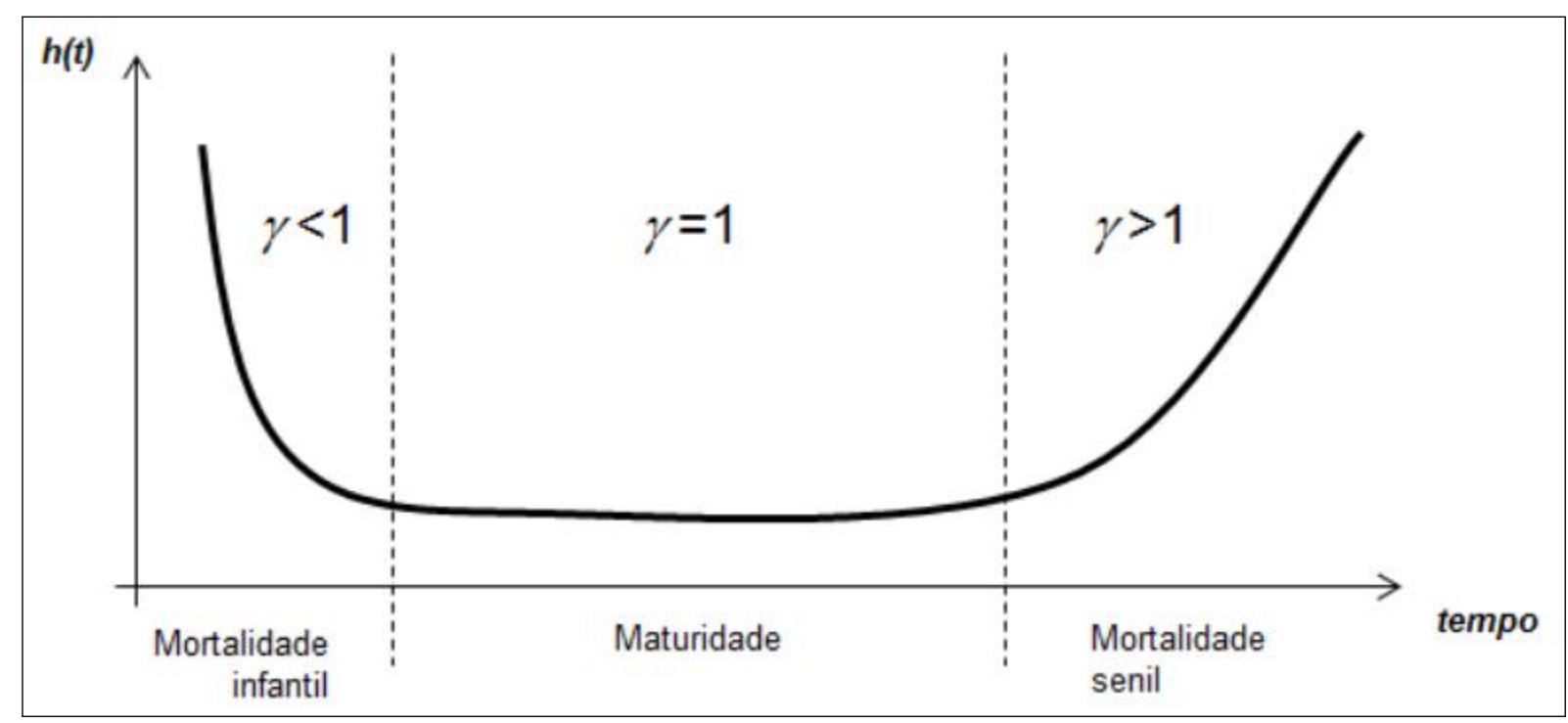

Fonte:Adaptado de Lafraia (2001) e Sellitto (2005).

\section{RESULTADOS E DISCUSSÕES}

Alguns gráficos relativos ao conjunto de dados foram construídos. Na Figura 2 está representado o histograma das frequências de falhas apresentadas na Tabela 1. 


\section{Figura 2 - Histograma de frequência das falhas}

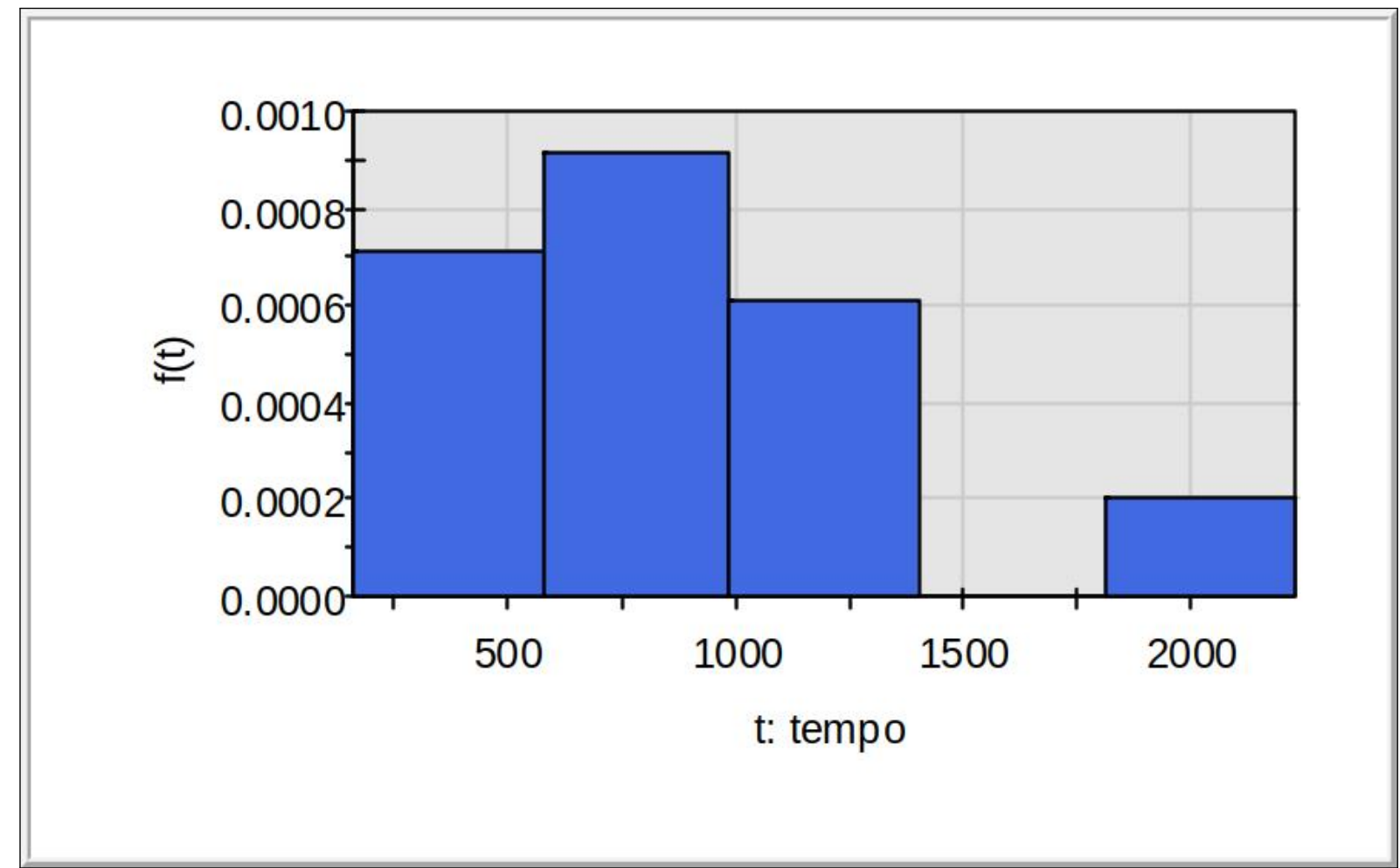

Fonte: Adaptado do ProConf (2019).

O formato do histograma pode ser associado aos gráficos da função densidade de probabilidade das distribuições Lognormal e Weibull, o que torna as duas candidatas a modelar adequadamente o conjunto de dados estudado. Na Figura 3 tem-se o papel de probabilidade correspondente das duas distribuições obtidas por meio do ProConf.

Figura 3 - Papel de probabilidade das distribuições Lognormal (esquerda) e Weibull (direita

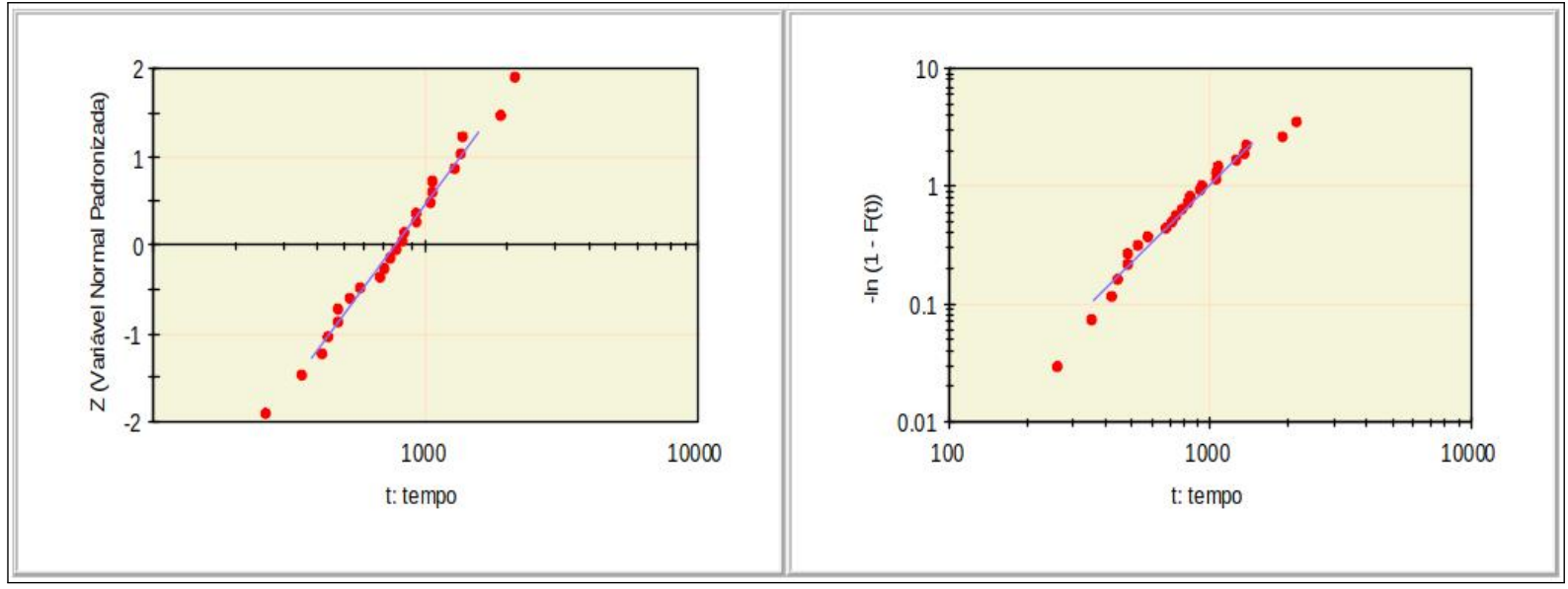

Fonte: Adaptado do ProConf (2019).

A análise dos gráficos de papel de probabilidade indica que ambas distribuições apresentam uma linha de tendência, ou seja, se ajustam perfeitamente em torno da reta, sem maiores dispersões. Dessa forma, não é possível definir visualmente o modelo que melhor se ajusta ao 
conjunto amostral.

Para uma análise mais precisa, serão utilizados os métodos analíticos, os testes de aderência Qui-Quadrado $\left(\mathrm{X}^{2}\right)$ e do Zolmogorov-Smirnov (K-S). Na Tabela 2 estão apresentados os valores-p para esses testes para as distribuições implementadas no ProConf.

Tabela 2 - Valor-p dos testes de aderência

\begin{tabular}{c|c|c|c}
\hline Modelo & $\mathrm{X}^{2}$ & $\mathrm{~K}-\mathrm{S}$ & Decisão \\
\hline Exponencial & 0,0100 & 0,0001 & Rejeitada \\
\hline Gama & 0,2152 & 0,3457 & Não pode ser rejeitada \\
\hline Lognormal & 0,2388 & 0,3979 & Não pode ser rejeitada \\
\hline Weibull & 0,1441 & 0,3033 & Não pode ser rejeitada \\
\hline
\end{tabular}

Fonte: Criada pelos autores com dados extraídos do ProConf (2019).

Para cada teste, o software informa o valor $\mathrm{p}$ e aponta quais distribuições podem ser rejeitadas. Observa-se a rejeição da hipótese de que a distribuição Exponencial modelasse a amostra de dados. Apesar de três distribuições não terem sido rejeitadas, o presente estudo utilizará a distribuição Weibull para a modelagem do tempo até a falha do transístor.

Fogliato e Ribeiro (2009) afirmam que para amostras de tempos até a falha de tamanho pequeno, que é o caso, supor dados seguindo uma distribuição de Weibull costuma ser um bom ponto de partida na análise. Além disso, a distribuição Weibull tem a vantagem de ser mais flexível no ajuste de diferentes tipos de dados, e permitir relacionar o seu parâmetro de forma à fase da curva da banheira (SELLITTO, 2005).

Com a definição do m odelo a s er s eguido foi possível e stimar s eus p arâmetros, bem como outros resultados dos ajustes apresentados pelo ProConf, conforme Tabela 3. O t10 e o t50 correspondem, respectivamente, aos valores limites de tempos nos quais $10 \%$ e $50 \%$ das falhas ocorreram. São apresentadas também as estimativas dos parâmetros da Weibull, representados por $\gamma$ e $\theta$.

\section{Tabela 3 - Resultados dos ajustes}

\begin{tabular}{c|c}
\hline Parâmetros & Resultados \\
\hline $\mathrm{t}_{10}$ (horas) & 310,51 \\
\hline $\mathrm{t}_{50}$ (horas) & 816,48 \\
\hline MTTF(horas) & 873,82 \\
\hline$\gamma$ & 2,07 \\
\hline$\theta$ & 998,65
\end{tabular}

Fonte: Criada pelos autores com dados extraídos do ProConf (2019).

Observa-se que o tempo médio até a falha dos transístores é de 873,82 horas. Metade dos componentes falham antes de atingirem 817 horas. Os parâmetros de forma e escala da distribuição Weibull foram, respectivamente, 2,07 e 998,65.

Segundo Sellitto (2005), o comportamento da taxa de falha de um equipamento ao longo do tempo pode ser analisado pela curva da banheira, que representa genericamente a função de risco $h(t)$ ao longo do ciclo de vida. A curva da banheira apresenta três períodos característicos 
de vida de componentes e equipamentos: mortalidade infantil, fase de maturidade, e mortalidade senil. Cada fase da curva está associada ao parâmetro de forma da distribuição Weibull, respectivamente, menor que 1 , igual a 1 e maior que 1 . Com base nos valores, pode-se assumir que a fase de vida da amostra dos transístores estudados corresponde à mortalidade senil, já que o parâmetro $\gamma$ foi maior que 1 .

As funções de confiabilidade e de risco modeladas segundo a distribuição Weibull estão representadas na Figura 4.

Figura 4 - Gráficos das funções de confiabilidade (esquerda) e de risco (direita)

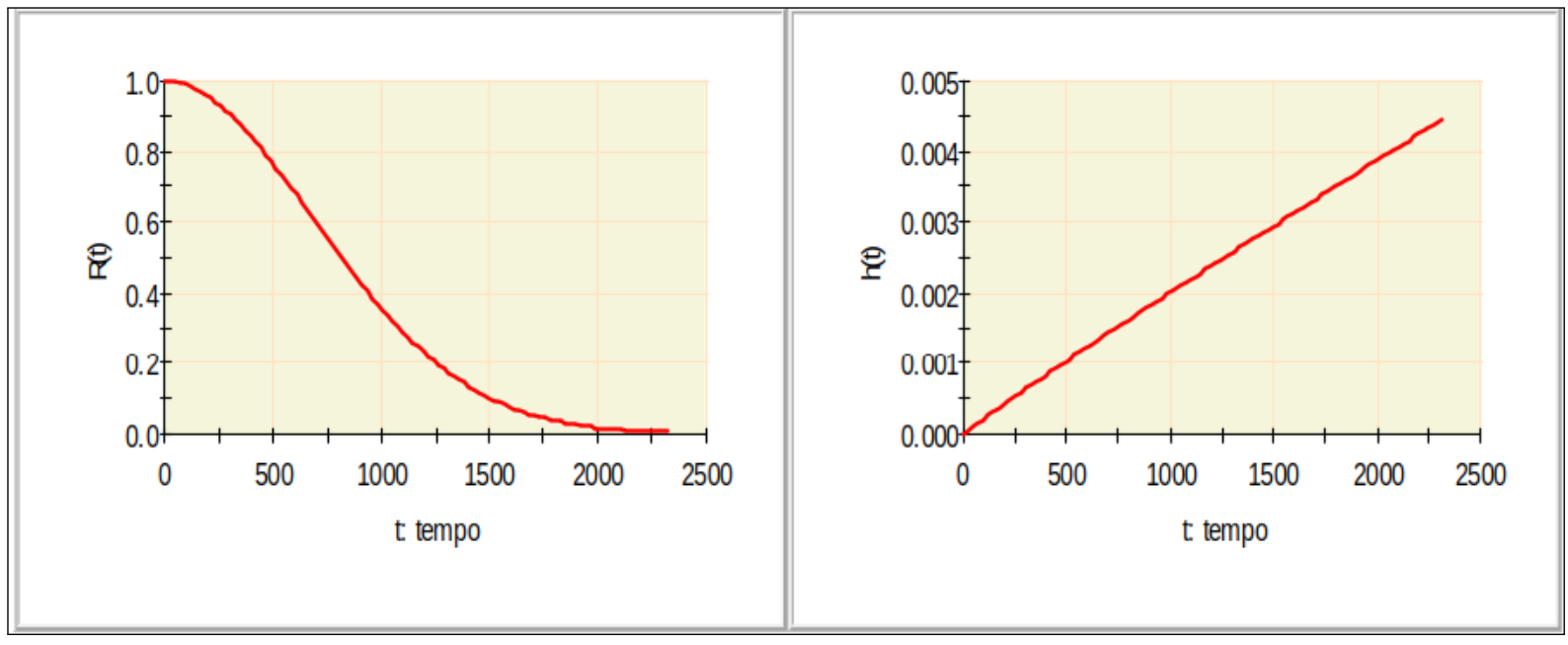

Fonte:Adaptado do ProConf (2019).

Observa-se que a confiabilidade sempre será decrescente com o tempo, já que as probabilidades de perfeita operação decaem conforme ocorra a utilização e o desgaste do equipamento. Pode-se observar também que a taxa de falha ou de risco é crescente, cuja origem é o próprio desgaste do equipamento, na fase final de mortalidade senil. Nessa fase, as falhas tendem a ter defeitos cumulativos. $\mathrm{O}$ aumento muito rápido da taxa de falha, normalmente fundamenta o critério de quando as peças devem ser substituídas e também determina a vida útil do produto ou sistema.

\section{CONCLUSÕES}

Neste estudo de confiabilidade foram utilizados dados reais com o equipamento em regime normal de trabalho. A análise dos tempos até a falha por meio de métodos gráficos e analíticos permitiu definir que a distribuição Weibull se adequa melhor aos dados, sendo possível obter as medidas de confiabilidade do tempo médio até a falha, os valores limites de tempo em que $10 \%$ e $50 \%$ das falhas ocorreram, bem como estimar os parâmetros da distribuição ajustada. $\mathrm{O}$ valor do parâmetro de forma obtido mostra que o comportamento do transístor se localiza na terceira fase da curva da banheira, ou seja, da mortalidade senil, indicando desgaste, taxa de risco crescente e confiabilidade decrescente.

O estudo da confiabilidade é de suma importância dentro de um processo industrial, 
pois o resultado obtido permite que a empresa reduza custos com mão de obra, não perca tempo de produção com a substituição de equipamentos, possibilitando um melhor funcionamento da empresa de forma geral. 


\section{REFERÊNCIAS}

ABNT. NBR 5462: Confiabilidade e mantenabilidade. Rio de Janeiro, 1994.

BRANDÃO, Mariane Olivier; ANDRADE, Paulo César de Resende. Modelagem dos dados de falhas de um pasteurizador de garrafas de cerveja. Revista de Engenharia e Tecnologia, v. 10, n. 2, p. 172-181, 2018.

CHIQUITO, Adenilson José; JúNIOR, Francesco Lanciotti. O transistor, 50 anos. Revista Brasileira de Ensino de Física, v. 20, n. 4, p. 309-314, 1998.

COLOSIMO, Enrico Antônio; FREITAS, Marta Afonso. Confiabilidade: análise de tempo de falha e testes de vida acelerados. Belo Horizonte: Escola de Engenharia da UFMG/Fundação Christiano Ottoni, 1997. 309 p.

CRUZ, Carlos Henrique Brito; PACHECO, Carlos Américo. Conhecimento e inovação: desafios do brasil no século xxi. Inovação Unicamp, Seção Íntegras, Campinas, v. 30, p. 12, 2004. Disponível em: <https://www.ifi.unicamp.br/ brito/artigos/inte-pacheco-brito.pdf>.

ELSAYED, Elsayed A. Reliability engineering. 1. ed. Reading, MA: Addison Wesley Longman, Inc., 1996.

FOGLIATO, Flavio; RIBEIRO, José Luis Duarte. Confiabilidade e manutenção industrial. Rio de Janeiro: Elsevier, 2009.

FRITSCH, Celso; RIBEIRO, José. Proconf: Um software orientado para análises de confiabilidade. In: XVIII Encontro Nacional de Engenharia de Produção. [S.l.: s.n.], 1998.

GAIO, Evandro Dias. Proposta de um plano de manutenção de um equipamento industrial através da utilização de ferramentas da manutenção centrada em confiabilidade. 2016. 66 p. Monografia (Bacharelado em Engenharia Mecânica) — Universidade Federal de Juiz de Fora, Juiz de Fora.

HAVIARAS, Gilberto Jorge. Metodologia para análise de confiabilidade de pneus radiais em frotas de caminhões de longa distância. 2005. 124 p. Dissertação (Mestrado em Engenharia Automotiva) - Escola Politécnica da Universidade de São Paulo, São Paulo.

KARDEC, Allan; NASCIF, Júlio. Manutenção-função estratégica. 3. ed. Rio de Janeiro: Qualitymark Editora Ltda, 2009.

KHAN, M. Shuaib; PASHA, G.R.; PASHA, Ahmed Hesham. Theoretical analysis of inverse Weibull distribution. WSEAS Transactions on Mathematics, Citeseer, v. 7, n. 2, p. 30-38, 2008.

LAFRAIA, João Ricardo Barusso. Manual de confiabilidade, mantenabilidade e disponibilidade. Rio de Janeiro: Qualitymark Editora Ltda, 2001.

LEEMIS, Lawrence Mark. Reliability: Probabilistic models and statistical methods. New Jersey: Prentice-Hall, 1995.

MENDES, Angélica Alebrant. Manutenção centrada em confiabilidade: uma abordagem quantitativa. 2011. 85 p. Dissertação (Mestrado em Engenharia de Produção) — Escola de Engenharia, Universidade Federal do Rio Grande do Sul, Porto Alegre.

PIAZZA, Gilberto. Introdução à engenharia da confiabilidade. EDUCS, Caxias do Sul, 2000. 
SELLITTO, Miguel Afonso. Formulação estratégica da manutenção industrial com base na confiabilidade dos equipamentos. Production, SciELO Brasil, v. 15, n. 1, p. 44-59, 2005.

SILVA, Victor Andrei. Administração da Produção: Análise do processo de produção de uma empresa de colchão. 2012. Disponível em: <http://www.administradores.com.br/artigos/ marketing/administracao-da-producao-analise-do-processo-de-producao-de-uma-empresade-colchao/64556/>. Acesso em: 29 jan. 2019. 